DESY 09-026

September 2009

\title{
Light Moduli in Almost No-Scale Models
}

\author{
Wilfried Buchmüller, Jan Möller and Jonas Schmidt \\ Deutsches Elektronen-Synchrotron DESY, Hamburg, Germany
}

\begin{abstract}
We discuss the stabilization of the compact dimension for a class of five-dimensional orbifold supergravity models. Supersymmetry is broken by the superpotential on a boundary. Classically, the size $L$ of the fifth dimension is undetermined, with or without supersymmetry breaking, and the effective potential is of no-scale type. The size $L$ is fixed by quantum corrections to the Kähler potential, the Casimir energy and Fayet-Iliopoulos (FI) terms localized at the boundaries. For an FI scale of order $M_{\mathrm{GUT}}$, as in heterotic string compactifications with anomalous $\mathrm{U}(1)$ symmetries, one obtains $L \sim 1 / M_{\mathrm{GUT}}$. A small mass is predicted for the scalar fluctuation associated with the fifth dimension, $m_{\rho} \lesssim m_{3 / 2} /(L M)$.
\end{abstract}




\section{Introduction}

Higher-dimensional supergravity and superstring theories provide a promising framework for the unification of matter, gauge interactions and gravity [1]. These theories possess vacua with unbroken supersymmetry and flat higher-dimensional Minkowski space. It is a challenging task to find four-dimensional non-supersymmetric locally stable Minkowski or de Sitter vacua, with compact extra dimensions smaller than the electroweak scale.

Classically, size and shape of the compact dimensions are generically undetermined. Stabilization can occur as a result of quantum corrections. In field theory, these include the Casimir energy [2-6] and localized Fayet-Iliopoulos (FI) terms [7]. Loop- and $\alpha^{\prime}$-corrections play a crucial role for the stabilization of volume moduli in string theory [8-11], in addition to fluxes. Furthermore, in string theory and field theory nonperturbative corrections to the superpotential are often required to achieve a complete stabilization of the compact dimensions $[12,13]$.

Recently, it has been suggested that the interplay of Casimir energy and localized FayetIliopoulos terms can lead to the stabilization of the compact dimensions [6]. For a FI mass scale $\mathcal{O}\left(M_{\mathrm{GUT}}\right)$, as it occurs in some compactifications of the heterotic string [14], one then obtains for the size of the compact dimensions $L \sim 1 / M_{\mathrm{GUT}}$. The height of the barrier which separates four-dimensional from ten-dimensional Minkowski space is $\mathcal{O}\left(m_{3 / 2}^{2} M_{\mathrm{GUT}}\right)$. It therefore vanishes for unbroken supersymmetry.

In this paper we study the interplay of supersymmetry breaking and FI terms in supergravity. We shall consider the simplest case of five-dimensional orbifold models, which include the dynamics of the radion superfield (cf. $[13,15,16])$. Such models can be considered a toy version for anisotropic compactifications of ten-dimensional string theories, which have one 'large' compact dimension. Due to the no-scale structure of the Kähler potential, it is impossible to realize non-supersymmetric locally stable Minkowski or de Sitter vacua at tree level [17-19]. The radion flat direction needs to be lifted by quantum corrections to the Kähler potential, which always include the Casimir energy.

As we shall see, perturbative corrections to the Kähler potential, together with a nonzero brane superpotential, imply 'almost no-scale' models, similar to the one proposed by Luty and Okada [20]. The chiral superfield, which generates the expectation value of the superpotential, couples to bulk fields. This coupling leads to a contribution to the radion potential, which is of the same order of magnitude as the Casimir energy. The resulting radion potential allows for metastable Minkowski or de Sitter vacua, without the need of an additional 'uplifting' mechanism.

The paper is organized as follows. Section 2 describes the no-scale model of a radion field coupled to a brane localized chiral superfield. The general structure of 'almost noscale' models is analyzed in Section 3, where also a formula for the radion mass is derived. The stabilization induced by localized FI terms is worked out in Section 4, which is followed by a brief summary in Section 5 . 


\section{A class of no-scale models}

Consider the bosonic part of the action of five-dimensional (5D) $\mathcal{N}=1$ supergravity compactified on $S_{1} / \mathbb{Z}_{2}$, with bulk and brane contributions

$$
S_{5}=S_{\text {bulk }}+\delta(y) S_{\text {vis }}+\delta(y-L) S_{\text {hid }},
$$

where

$$
S_{\text {bulk }}=\frac{M_{5}^{3}}{2} \int d^{4} x \int_{0}^{L} d y \sqrt{-g_{5}}\left\{R_{5}-\frac{1}{2} H^{M N} H_{M N}+\mathcal{L}_{\text {bulk }}\right\} .
$$

Here $H_{M N}=\partial_{M} B_{N}-\partial_{N} B_{M}$ is the field strength of the graviphoton, the spin-1 component of the supergravity multiplet. Dimensional reduction of this action on the background metric

$$
d s_{5}^{2}=g_{\mu \nu}(x) d x^{\mu} d x^{\nu}+r^{2}(x) d y^{2}
$$

leads to

$$
S_{4}=\frac{M^{2}}{2} \int d^{4} x \sqrt{-g} r\left\{R-\frac{1}{r^{2}} \partial_{\mu} B_{5} \partial^{\mu} B_{5}+\mathcal{L}_{\text {bulk }}^{(4)}\right\}+S_{\text {branes }}\left[g^{\mu \nu}\right],
$$

where we have only kept $g_{\mu \nu}, g_{55}$ and $B_{5}$, which have even $\mathbb{Z}_{2}$ parity. The remaining fields $g_{\mu 5}, B_{\mu}$ are $\mathbb{Z}_{2}$ odd and thus do not have light modes. $M=\sqrt{M_{5}^{3} L}$ is the 4 D Planck mass, for a stabilized radion with $r_{0}=1$ in the vacuum. Note that the radion field, i.e., the scale factor of the fifth dimension, is dimensionless and has no quadratic kinetic term. Due to the bulk-brane structure, $r$ couples non-universally to the matter sector, hence it is not a Brans-Dicke scalar.

After a conformal transformation of the metric, $g_{\mu \nu} \rightarrow r^{-1} g_{\mu \nu}$, one finds for the action in the Einstein frame,

$$
\begin{array}{r}
S_{4}=\frac{M^{2}}{2} \int d^{4} x \sqrt{-g}\{R \\
-\frac{3}{2 r^{2}} g^{\mu \nu} \partial_{\mu} r \partial_{\nu} r-\frac{1}{r^{2}} \partial_{\mu} B_{5} \partial^{\mu} B_{5} \\
\left.+\frac{1}{r} \mathcal{L}_{\text {bulk }}^{(4)}\left[r g^{\mu \nu}\right]\right\}+S_{\text {branes }}\left[r g^{\mu \nu}\right] .
\end{array}
$$

This action contains a quadratic kinetic term for the radion field. Note the presence of the unusual factor 3, which will reappear in the Kähler potential below. This factor indicates that the kinetic term is solely generated by the conformal transformation.

A globally supersymmetric theory is characterized by a holomorphic superpotential $W(z)$ and a real function $\Omega(z, \bar{z})$ which yields the kinetic terms

$$
\mathcal{L}_{\text {kin }}^{\text {global }}=\Omega_{i \bar{j}} \partial_{\mu} z^{i} \partial^{\mu} z^{\bar{j}}, \quad \Omega_{i \bar{j}} \equiv \partial_{i} \partial_{\bar{j}} \Omega .
$$

In the corresponding supergravity theory kinetic terms and scalar potential are determined by the Kähler potential

$$
K=-3 M^{2} \ln \left(-\frac{\Omega}{3 M^{2}}\right),
$$


with

$$
\mathcal{L}_{\text {kin }}^{\text {local }}=K_{i j} \partial_{\mu} z^{i} \partial^{\mu} \bar{z}^{\bar{j}}
$$

and

$$
V_{F}=e^{K / M^{2}}\left[\left(W_{i}+M^{-2} K_{i} W\right) K^{i \bar{j}}\left(\bar{W}_{\bar{j}}+M^{-2} K_{\bar{j}} \bar{W}\right)-3 M^{-2}|W|^{2}\right] .
$$

Let us now consider a model with minimal field content and include one brane chiral superfield $X$ with canonical kinetic term, such that

$$
\Omega=-\frac{3 M^{2}}{2}(T+\bar{T})+X \bar{X}
$$

The Einstein frame component action (2.5) is then obtained for the Kähler potential [21]

$$
K=-3 M^{2} \ln \left(\frac{T+\bar{T}}{2}-\frac{X \bar{X}}{3 M^{2}}\right) .
$$

The scalar component of the radion superfield contains the brane field $X$,

$$
T=r+\frac{X \bar{X}}{3 M^{2}}+i \sqrt{\frac{2}{3}} B_{5},
$$

compensating for the non-diagonal entries in the Kähler metric.

The Kähler potential (2.11) has no-scale structure [22],

$$
K^{i} K_{i}=3 M^{2}
$$

which is characteristic for a universal Kähler modulus. Hence, the negative-definite contribution to the scalar potential vanishes, and one obtains

$$
V_{F}=\frac{1}{r^{2}} W_{X} \bar{W}_{\bar{X}}
$$

The equations of motion

$$
\partial_{r} V_{F}=0, \quad \partial_{X} V_{F}=0
$$

are simultaneously satisfied at stationary points of the superpotential,

$$
\left.\partial_{X} W\right|_{X_{0}}=0 \text {. }
$$

The potential then vanishes for all values of $r$, satisfying the Minkowski condition $V_{F}=0$, and the size of the compact dimension is undetermined (cf. Fig. 1).

The Kähler potential does not depend on $B_{5}$, the imaginary part of the complex scalar $T$. At minima of the superpotential $W$ also the radion is a flat direction. Hence, the corresponding two scalar masses vanish,

$$
M_{1}^{2}=0, \quad M_{2}^{2}=0,
$$




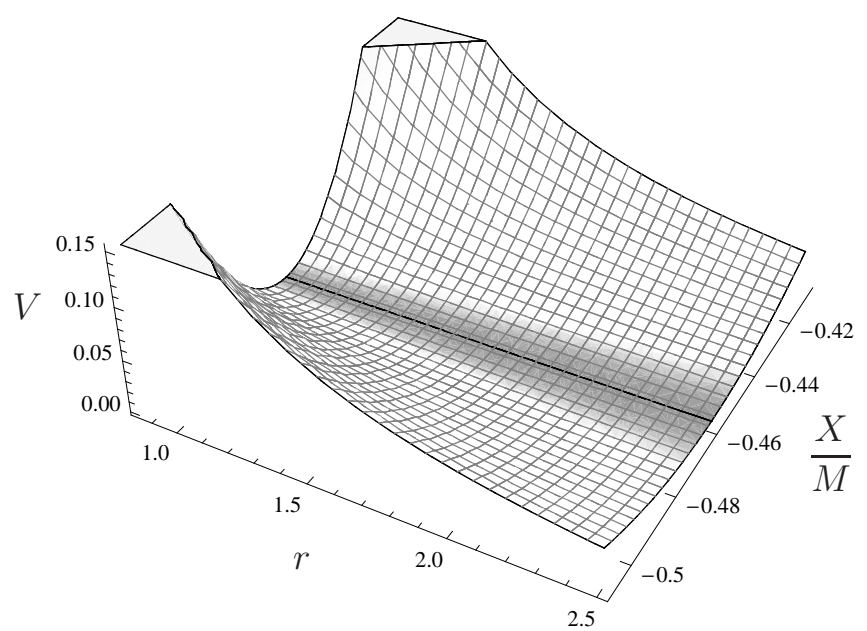

Figure 1: Example of a no-scale potential, in units of $m_{3 / 2}^{2} M^{2}$. It follows from the quadratic superpotential (4.22) for the choice $\sqrt{\sigma_{0}} \simeq 0.46$.

whereas the masses of real and imaginary part of $X$ are equal and positive,

$$
M_{3}^{2}=M_{4}^{2}=\frac{1}{4} W_{X X} \bar{W}_{\bar{X} \bar{X}} .
$$

In the limit $\left|W_{X X}\right| \rightarrow \infty$, the degrees of freedom of $X$ decouple, and it becomes a spectator field, i.e., only its vacuum expectation value (VEV) is relevant.

For non-vanishing superpotential, supersymmetry is spontaneously broken. The fermionic component of the radion superfield acts as the goldstino. The gravitino mass is given by

$$
m_{3 / 2}^{2}=e^{K / M^{2}} \frac{|W|^{2}}{M^{4}}=r^{-3} \frac{|W|^{2}}{M^{4}} .
$$

As expected in no-scale models, the gravitino mass 'slides' with the expectation value of the radion field.

The potential depicted in Fig. 1 illustrates the continuous vacuum degeneracy which is generic for no-scale models. It is well known that Kähler potentials of the type $K=-3 M^{2} \ln r$ do not admit non-supersymmetric Minkowski vacua with a positive definite mass matrix [17-19]. A necessary condition for the latter can be formulated as [18]

$$
R_{i \bar{j} k l} G^{\bar{l}} G^{k} G^{\bar{j}} G^{i}<6 M^{2},
$$

where $R_{i \bar{j} k \bar{l}}$ denotes the Riemann curvature of the Kähler manifold, and

$$
G=K+M^{2} \ln \frac{|W|^{2}}{M^{6}} .
$$

The scalar potential is then given by

$$
V=m_{3 / 2}^{2}\left(G^{i} G_{i}-3 M^{2}\right) \text {. }
$$


For the two-field no-scale Kähler potential (2.11) vanishing of the vacuum energy implies

$$
R_{i \bar{j} k \bar{l}} G^{\bar{l}} G^{k} G^{\bar{j}} G^{i}=6 M^{2}
$$

This result holds for any superpotential $W(X, T)$, even in the presence of nonperturbative corrections. Therefore at least one flat direction is unavoidable 1 We conclude that loop corrections are crucial for the stabilization of the radion in a Minkowski vacuum.

\section{Almost no-scale models}

Quantum corrections change the real function $\Omega_{0}$ of no-scale models to

$$
\Omega=\Omega_{0}+\Delta \Omega
$$

where

$$
\Omega_{0}=-3 M^{2}\left(\frac{T+\bar{T}}{2}-\frac{\sigma}{3}\right), \quad \Delta \Omega \equiv 3 M^{2} r \kappa(r, \sigma), \quad \sigma \equiv \frac{X \bar{X}}{M^{2}} .
$$

The corresponding Kähler potential is given by

$$
\begin{aligned}
K & =-3 M^{2} \ln \left[-\Omega_{0}\left(1+\frac{\Delta \Omega}{3 M^{2} \Omega_{0}}\right)\right] \\
& =-3 M^{2}\left[\ln \left(\frac{T+\bar{T}}{2}-\frac{\sigma}{3}\right)+\ln (1-\kappa)\right] .
\end{aligned}
$$

In the following we shall analyze the effect of the correction $\kappa$, which turns the no-scale model of the previous section into an almost no-scale model.

It is straightforward to calculate the $\mathcal{O}(\kappa)$ correction to the no-scale potential,

$$
\begin{aligned}
V_{F}= & \frac{1}{r^{2}} W_{X} \bar{W}_{\bar{X}}\left(1+2 \kappa+\partial_{r}(r \kappa)-3 r \partial_{\sigma}\left(\sigma \partial_{\sigma} \kappa\right)\right) \\
& +\frac{3\left(X W_{X} \bar{W}+W \bar{X} \bar{W}_{\bar{X}}\right)}{M^{2} r^{2}} \partial_{r}\left(r \partial_{\sigma} \kappa\right)-\frac{3 W \bar{W}}{M^{2} r^{2}}\left(2 \partial_{r} \kappa+r \partial_{r}^{2} \kappa\right) .
\end{aligned}
$$

The tree level minimum $X_{0}$ is now shifted to $X_{0}+\Delta X$. At linear order in $\Delta X$, the extremum condition

$$
\left.\partial_{X} V_{F}\right|_{X_{0}+\Delta X}=0
$$

implies

$$
\Delta X=\left.\frac{3 W}{M^{2} W_{X X}}\left(-\bar{X} \partial_{r}\left(r \partial_{\sigma} \kappa\right)+\frac{\bar{W}}{M^{2} W_{X X}} X\left(2 \partial_{r} \partial_{\sigma} \kappa+r \partial_{r}^{2} \partial_{\sigma} \kappa\right)\right)\right|_{X_{0}, r_{0}} .
$$

\footnotetext{
${ }^{1}$ Note that this argument also applies to the mechanism of [13], where the $F$-term uplift induces a flat direction in the hidden sector.
} 
Our systematic expansion in $\kappa$ is consistent as long as $|\Delta X| /\left|X_{0}\right| \leq \mathcal{O}(\kappa)$. According to Eq. (3.6) this holds if $\left|W_{X X}\right| \geq \mathcal{O}\left(|W| / M^{2}\right)$, i.e.,

$$
M_{3,4} \geq \mathcal{O}\left(m_{3 / 2}\right) \text {. }
$$

Note that the corresponding fermion mass has to satisfy the same bound.

The resulting leading order effective potential can then be read off from Eq. (3.4),

$$
V^{(1)}(r, \sigma)=-\frac{3|W|^{2}}{M^{2}}\left(\frac{2}{r^{2}} \partial_{r} \kappa(r, \sigma)+\frac{1}{r} \partial_{r}^{2} \kappa(r, \sigma)\right) .
$$

The stabilization of the radion at $r_{0}$ leads to a mass term for the corresponding scalar fluctuations,

$$
r=r_{0}+\delta r=1+\sqrt{\frac{2}{3}} \rho,
$$

where the definition of $\rho$ renders a canonical kinetic term. The mass matrix of the complex scalars $T$ and $X$ has the eigenvalues

$$
\begin{aligned}
& M_{1}^{2}=0, \quad M_{2}^{2}=\left.\frac{|W|^{2}}{M^{4}}\left(4 \partial_{r}^{3} \kappa+\partial_{r}^{4} \kappa\right)\right|_{X_{0}, r_{0}}+\mathcal{O}\left(\kappa^{2} m_{3 / 2}^{2}\right) \\
& M_{3}^{2}=M_{4}^{2}=\left.\frac{1}{4} W_{X X} \bar{W}_{\bar{X} \bar{X}}\right|_{X_{0}}+\mathcal{O}\left(\kappa m_{3 / 2}^{2}\right) .
\end{aligned}
$$

We conclude that the radion mass is $\mathcal{O}(\kappa)$ relative to the gravitino mass 2 Note that this result does not depend on details of the stabilization mechanism. It is unavoidable whenever the vacuum is stabilized by quantum corrections to the Kähler potential, which can be treated perturbatively.

\section{Perturbative stabilization of the radion}

In the previous section we discussed how quantum corrections deform the no-scale Kähler potential such that a stable, non-supersymmetric Minkowski vacuum can emerge. We shall now present a specific example where the quantum corrections leading to Casimir energy and localized Fayet-Iliopoulos terms are taken into account. In terms of these corrections the size $L$ of the extra dimension can be explicitly calculated.

In general, there is a contribution to $\kappa$ from the Casimir energy of the gravitational multiplet [23] and other massless bulk fields,

$$
\Delta \Omega_{\mathrm{C}}(r)=-\frac{1}{2 L^{2}}\left(A r^{3}+3 B r^{2}+\frac{C}{r^{2}}\right) \equiv 3 M^{2} r \kappa_{\mathrm{C}}(r),
$$

which, according to (3.8), corresponds to the potential

$$
V_{\mathrm{C}}^{(1)}(r)=\frac{3|W|^{2}}{M^{4} L^{2} r^{2}}\left(A r+B+\frac{C}{r^{4}}\right) .
$$

\footnotetext{
${ }^{2}$ In the case of $\alpha^{\prime}$-corrections, a similar relation for the radion mass has been obtained in [19].
} 
The Casimir energy (4.2) vanishes for $W=0$, i.e., for unbroken supersymmetry. The constant $C$ is determined by the number of massless degrees of freedom in the bulk, the constants $A$ and $B$ are bulk and brane tensions, respectively. They are needed for the renormalization of the divergent Casimir energy and depend on the renormalization scale (cf. $[4,6]$ ). These constants have been used to stabilize the radion at a minimum with vanishing cosmological constant [3] 3 3 Our expansion around no-scale models is consistent as long as $A$ and $B$ are $\mathcal{O}(\kappa)$. For simplicity, we choose $A=B=0$ in the following. As we shall see, radion stabilization in a Minkowski vacuum can still be achieved by fine tuning the remaining parameters of the scalar potential.

In addition, massive bulk fields contribute to the Casimir energy. The resulting term in the effective radion potential is known to take the form $[3,20]$

$$
\begin{aligned}
V_{\mathrm{C}^{\prime}}^{(1)}(r)=\frac{3|W|^{2}}{M^{4} L^{2} r^{2}} \frac{C^{\prime}}{r^{4}}( & \frac{M_{\mathrm{bulk}}^{2} L^{2} r^{2}}{3} \operatorname{Li}_{1}\left(e^{-M_{\mathrm{bulk}} L r}\right) \\
& \left.+M_{\mathrm{bulk}} L r \operatorname{Li}_{2}\left(e^{-M_{\mathrm{bulk}} L r}\right)+\operatorname{Li}_{3}\left(e^{-M_{\mathrm{bulk}} L r}\right)\right),
\end{aligned}
$$

with the polylogarithmic functions

$$
\operatorname{Li}_{s}\left(e^{-M_{\mathrm{bulk}} L r}\right) \equiv \sum_{k=1}^{\infty} \frac{e^{-k M_{\mathrm{bulk}} L r}}{k^{s}} .
$$

The constant $C^{\prime}$ in (4.3) is related to the number of degrees of freedom with mass $M_{\text {bulk }}$, and will be specified below. Note that $\kappa_{\mathrm{C}^{\prime}}(r)$ can be obtained by integrating Eq. (3.8) for the potential (4.3) (cf. [23]), which, however, is not required for our further calculations.

There are further corrections to the potential in the presence of brane-localized kinetic terms. Their contribution corresponds to a two-loop effect [5,25] and is therefore subleading. Moreover, in string theory the Kähler potential is modified by supersymmetric loop corrections and $\alpha^{\prime}$-corrections, which could be treated as additional contributions to the function $\kappa$.

In orbifold compactifications, generically Fayet-Iliopoulos terms of anomalous U(1) symmetries arise at fixed points $[7,14]$. They induce a non-trivial vacuum configuration of the scalar sector: Bulk fields that are charged under the U(1) symmetry develop vacuum expectation values and become massive. These VEVs ensure vanishing $F$ - and $D$-terms in the bulk and at the fixed points. In the simplest case of one hypermultiplet, containing the $\mathcal{N}=1$ chiral multiplets $H$ and $H^{c}$, one has

$$
\begin{aligned}
\Delta \Omega_{\mathrm{bulk}} & =H \bar{H}+H^{c} \bar{H}^{c}, \\
\Delta \Omega_{\mathrm{brane}} & =\frac{\lambda^{\prime}}{M_{5}^{3}}\left(H \bar{H}+H^{c} \bar{H}^{c}\right) X \bar{X} .
\end{aligned}
$$

A detailed analysis [26] shows that if the sum of the FI terms is non-zero, one of the two chiral multiplets, say $H$, develops an $r$-dependent $\mathrm{VEV}$, while $\left\langle H^{c}\right\rangle=0$. In the $4 \mathrm{D}$ theory

\footnotetext{
${ }^{3}$ Note, however, that the choice of the constants has to be consistent with supersymmetry [24].
} 


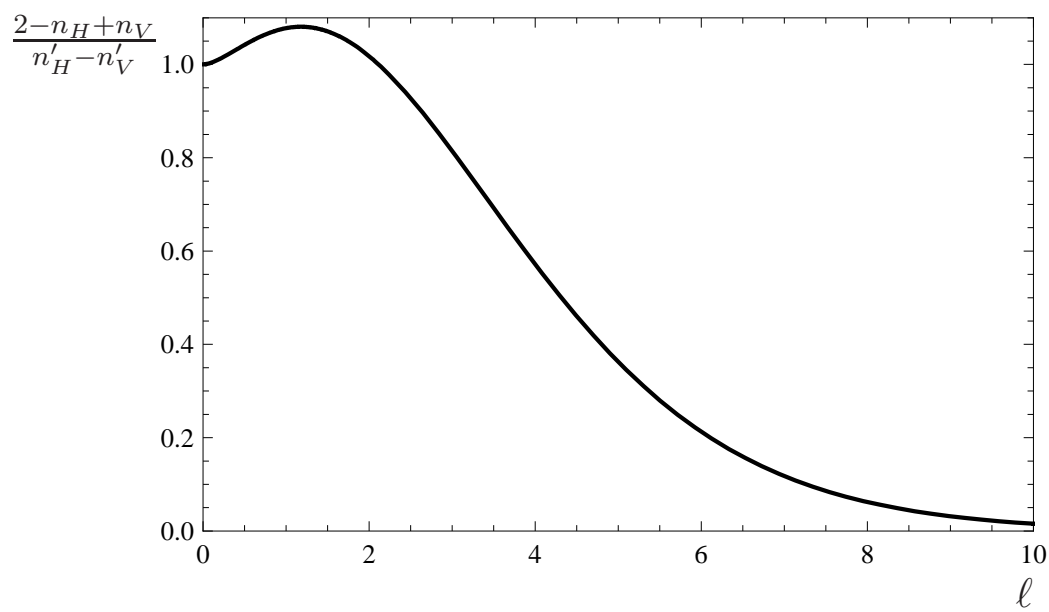

Figure 2: The relation between the bulk field content and the size of the compact dimension $L=\ell / M_{\text {bulk }}$. The plotted ratio of multiplicities has a maximum at $\hat{\ell} \simeq 1.2$.

one then obtains (cf. (3.2))

$$
\begin{aligned}
\Delta \Omega_{\mathrm{FI}} & =\int_{0}^{L} d y\left[r\langle H \bar{H}\rangle+\delta(y-L) \frac{\lambda^{\prime}}{M_{5}^{3}}\langle H \bar{H}\rangle X \bar{X}\right] \\
& =\xi\left(1+\frac{\lambda X \bar{X}}{M^{2} r}\right) \equiv 3 M^{2} r \kappa_{\mathrm{FI}}(r, \sigma) .
\end{aligned}
$$

Here $\xi$ is the sum of the two FI terms localized at the fixed points at $y=0$ and $y=L$, and $M_{5}^{3} L=M^{2}$, provided $r_{0}=1$. The different couplings $\lambda$ and $\lambda^{\prime}$ reflect the discrepancy between the condensate at $y=L$ and its average value. The function $\kappa_{\text {FI }}$ corresponds to the effective radion potential (cf. (3.8))

$$
V_{\mathrm{FI}}^{(1)}(r, \sigma)=-\frac{2 \lambda \sigma}{r^{3}} \frac{\xi|W|^{2}}{M^{4}} .
$$

Note that the $r$-dependent background field value results in a deformation of the KaluzaKlein spectrum. The special case $\xi=0$, accompanied by strong localization of the bulk fields, was discussed in [27]. Here we consider nearly constant VEVs. We then expect that the backreaction on the internal geometry remains negligible, such that the flat orbifold is a valid approximation. However, small warping could be treated as an additional contribution to the $\kappa$ correction (cf. [25] and references therein).

Furthermore, the VEV $\langle H\rangle$ breaks the anomalous $\mathrm{U}(1)$ and the corresponding gauge boson acquires a mass $M_{V}=\mathcal{O}(\sqrt{\xi})$, like the hyperscalars. For simplicity, we assume a common mass parameter for the $\mathrm{U}(1)$ vector- and massive hypermultiplets $\mathbb{A} \mathcal{O}(1)$ mass differences would not change our results qualitatively. With $\xi=\mathcal{O}\left(M_{\text {GUT }}^{2}\right)$ (cf. [14]), one has

$$
M_{H}=M_{V}=M_{\mathrm{bulk}}=\mathcal{O}\left(M_{\mathrm{GUT}}\right) .
$$

\footnotetext{
${ }^{4}$ Their contribution to the Casimir energy was neglected in [6].
} 


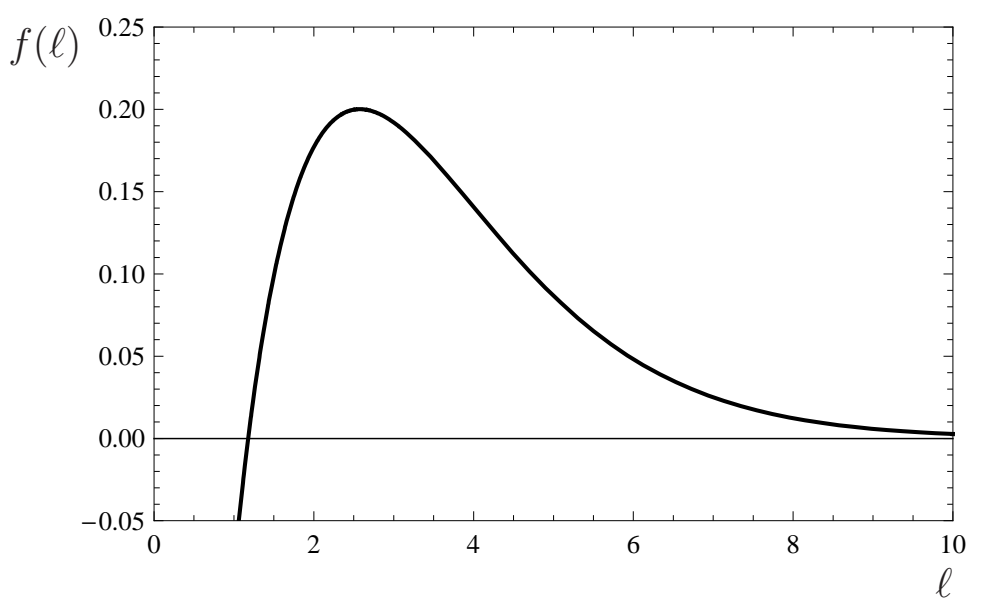

Figure 3: The function $f(\ell), \ell=L M_{\text {bulk }}$, which determines the radion mass $m_{\rho}(c f$. (4.18))).

In terms of the dimensionless parameter $\ell$ defined by

$$
L=\frac{\ell}{M_{\mathrm{bulk}}},
$$

the resulting radion effective potential reads up to terms $\mathcal{O}(\kappa)$,

$$
\begin{aligned}
V^{(1)}(r, \sigma)= & V_{\mathrm{FI}}^{(1)}(r, \sigma)+V_{\mathrm{C}}^{(1)}(r)+V_{\mathrm{C}^{\prime}}^{(1)}(r) \\
= & \frac{3|W|^{2}}{M^{2} r^{2}} \frac{M_{\text {bulk }}^{2}}{M^{2}}\left[-\frac{2 \lambda \sigma}{3 r^{3}} \frac{\xi}{M_{\text {bulk }}^{2}}+\frac{C}{\ell^{2} r^{4}}\right. \\
& \left.\quad+\frac{C^{\prime}}{\ell^{2} r^{4}}\left(\frac{\ell^{2} r^{2}}{3} \operatorname{Li}_{1}\left(e^{-\ell r}\right)+\ell r \operatorname{Li}_{2}\left(e^{-\ell r}\right)+\operatorname{Li}_{3}\left(e^{-\ell r}\right)\right)\right] .
\end{aligned}
$$

The constant $C\left(C^{\prime}\right)$ is determined by the number of massless (massive) vector and hypermultiplets $n_{V}, n_{H}\left(n_{V}^{\prime}, n_{H}^{\prime}\right)$, respectively,

$$
C=\frac{\zeta(3)}{32 \pi^{2}}\left(n_{H}-n_{V}-2\right), \quad C^{\prime}=\frac{1}{32 \pi^{2}}\left(n_{H}^{\prime}-n_{V}^{\prime}\right)
$$

In the minimal case $n_{H}=n_{V}=0$, only the supergravity multiplet contributes to the massless sector. Note that only hypermultiplets give rise to positive contributions, leading to repulsive behaviour at small distances. A local minimum can be obtained for $C<0$, $C^{\prime}>0$, and therefore

$$
n_{H}<n_{V}+2, \quad n_{H}^{\prime}>n_{V}^{\prime} .
$$

With $n_{H}^{\prime}-n_{V}^{\prime}=1 \ldots \mathcal{O}(100)$, as in heterotic orbifolds [28], this yields the parameter range $10^{-2} \lesssim C^{\prime} \lesssim 1$ 
We shall now show how to obtain a ground state with vanishing vacuum energy and determine the corresponding compactification scale. For this, we have to solve the equations

$$
\left.\partial_{r} V^{(1)}\right|_{r_{0}, \sigma_{0}}=0,\left.\quad V^{(1)}\right|_{r_{0}, \sigma_{0}}=0 .
$$

Imposing $r_{0}=1$, we obtain two conditions on the quantities $\ell$ and $\sigma_{0}$,

$$
\begin{aligned}
\frac{C}{C^{\prime}} & =\frac{\ell^{2}}{3}\left[\frac{\ell}{1-e^{\ell}}-2 \operatorname{Li}_{1}\left(e^{-\ell}\right)\right]-\ell \operatorname{Li}_{2}\left(e^{-\ell}\right)-\operatorname{Li}_{3}\left(e^{-\ell}\right), \\
\frac{\lambda \sigma_{0}}{C^{\prime}} & =\frac{M_{\text {bulk }}^{2}}{2 \xi}\left[\frac{\ell}{1-e^{\ell}}-\operatorname{Li}_{1}\left(e^{-\ell}\right)\right] .
\end{aligned}
$$

The RHS of (4.15) is negative and bounded from below, which translates into a condition on the field content (cf. Fig. 2),

$$
0<\frac{2-n_{H}+n_{V}}{n_{H}^{\prime}-n_{V}^{\prime}} \lesssim 1.1 .
$$

If this bound is satisfied, Eq. (4.15) can be solved for $\ell$. For local minima of the radion potential, this gives the size $L$ in units of $1 / M_{\text {bulk }}$ (cf. Eq. (4.10)).

Expanding the potential (4.11) around the local Minkowski vacuum and using (4.16), one obtains for the radion mass

$$
\frac{m_{\rho}^{2}}{m_{3 / 2}^{2}}=C^{\prime}\left(\frac{M_{\text {bulk }}}{M}\right)^{2} f(\ell),
$$

where

$$
f(\ell)=\frac{2}{3}\left[\frac{\ell\left(1+(\ell-1) e^{\ell}\right)}{\left(e^{\ell}-1\right)^{2}}-\operatorname{Li}_{1}\left(e^{-\ell}\right)\right] .
$$

The radion mass vanishes for $\ell=\hat{\ell} \simeq 1.2$, where the ratio $C / C^{\prime}$ is maximal (cf. Figs. 2,3). For $\ell>\hat{\ell}, m_{\rho}^{2}$ is positive and we have a stable Minkowski vacuum with $L \gtrsim 1 / M_{\text {GUT }}$ (cf. (4.9)). Fig. 3 also demonstrates that $f(\ell)$ has a local maximum, which yields an upper bound on the radion mass. For $C^{\prime} \lesssim 1$, one obtains

$$
\frac{m_{\rho}^{2}}{m_{3 / 2}^{2}} \lesssim 0.2\left(\frac{M_{\mathrm{bulk}}}{M}\right)^{2} \text {. }
$$

Larger radion masses require a huge number of massive species.

Having determined the size $L$ of the compact dimension by solving Eq. (4.15), we still have to satisfy Eq. (4.16). This is a condition on $\lambda \sigma_{0}$. Since the RHS of (4.16) is negative, the coupling $\lambda$ also has to be negative. Given $\lambda$, this yields a condition on the expectation value $\sigma_{0}=X_{0} \bar{X}_{0} / M^{2}$, and therefore on the parameters of the brane superpotential, which 


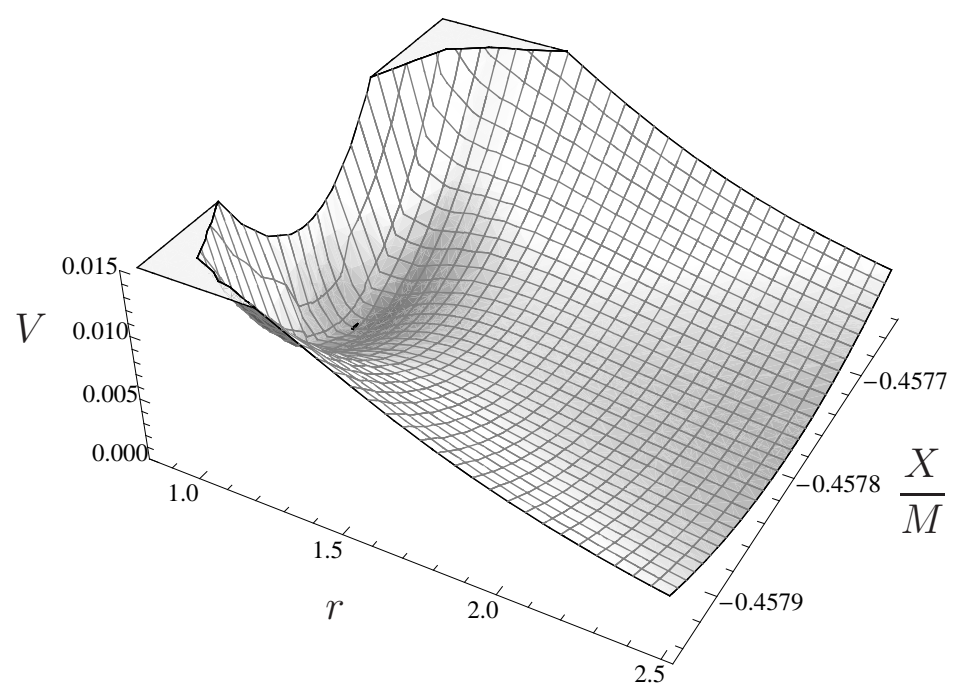

Figure 4: The two-field potential $V_{F}(X, r)$ given by (4.23) in units of $m_{3 / 2}^{2} M_{\text {bulk }}^{2}$, for the choice $\xi / M_{\text {bulk }}^{2}=1, \lambda=-1, C^{\prime} \simeq 1.0$ and $\ell \simeq 2.1$.

determine this VEV. This condition represents the fine tuning which is needed to obtain a Minkowski vacuum. With $M_{\text {bulk }}^{2} / \xi \simeq 1$, one obtains the upper bound

$$
|\lambda| \sigma_{0} \lesssim 0.4 C^{\prime}
$$

Hence, for $C^{\prime}<1$ and $|\lambda|=\mathcal{O}(1)$, the expectation value of $X$ is smaller than the Planck mass.

As an example, consider the superpotential

$$
W(X)=m_{3 / 2} M^{2}\left[2 \frac{X}{\sqrt{\sigma_{0}} M}+\left(\frac{X}{\sqrt{\sigma_{0}} M}\right)^{2}\right],
$$

which gives $X=\sqrt{\sigma_{0}} M$ up to terms $\mathcal{O}\left(\Delta X / \sqrt{\sigma_{0}}\right)$ (cf. 3.6). Note that Eq. (4.22) may represent the expansion of a nonperturbative brane superpotential up to second order in the field $X$. The corresponding two-field potential (3.4) is given by

$$
V_{F}(X, r)=\frac{1}{r^{2}} W_{X} \bar{W}_{\bar{X}}-\lambda \xi \frac{\left(X W_{X} \bar{W}+W \bar{X} \bar{W}_{\bar{X}}\right)}{M^{4} r^{4}}+V^{(1)}(r, \sigma)+\mathcal{O}\left(\left|W_{X}\right|^{2} \kappa\right)
$$

The potential is plotted in Fig. 4 in the vicinity of $X_{0}$, which clearly illustrates the almost no-scale structure of our model compared to the no-scale case shown in Fig. 1.

Figure 5 shows the resulting radion potential $V_{F}\left(X_{0}, r\right)$ for $\ell \simeq 2.1$, which corresponds to $n_{H}^{\prime}-n_{V}^{\prime}=2-n_{H}+n_{V}$. The stable Minkowski vacuum is separated from the runaway solution by a barrier of height

$$
V_{\text {barrier }} \ll m_{3 / 2}^{2} M_{\text {GUT }}^{2} .
$$




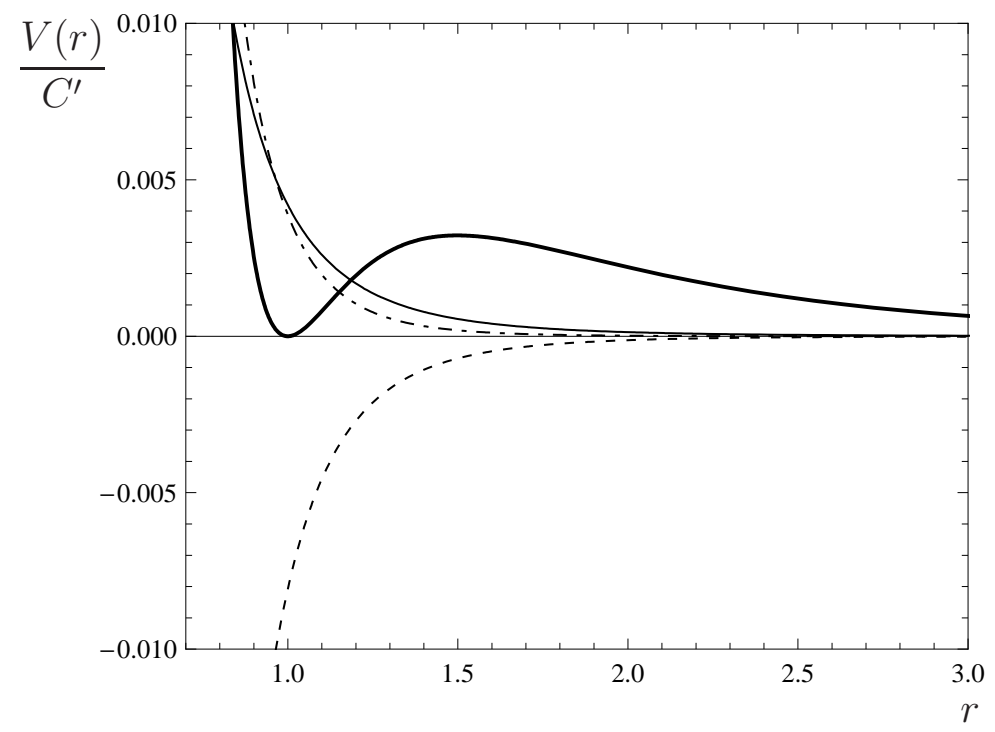

Figure 5: The radion effective potential $V(r) / C^{\prime}$ (bold curve), in units of $m_{3 / 2}^{2} M_{\text {bulk }}^{2}$ for $\ell \simeq 2.1$. The dashed (dot-dashed) and the thin curve are the contributions to the Casimir energy of massless (massive) bulk degrees of freedom and the FI-term induced contribution, respectively, scaled by a factor $1 /\left(100 C^{\prime}\right)$.

Vanishing of the vacuum energy in the local minimum requires a precise cancellation between three different contributions to the potential, all $\mathcal{O}\left(m_{3 / 2}^{2} M_{\text {GUT }}^{2}\right)$. One may also introduce a small positive vacuum energy, $\Lambda \sim\left(10^{-3} \mathrm{eV}\right)^{4}$, which would correspond to the fine tuning

$$
\frac{\Lambda}{m_{3 / 2}^{2} M_{\mathrm{GUT}}^{2}} \sim 10^{-90}
$$

for a gravitino mass $m_{3 / 2}=\mathcal{O}(\mathrm{TeV})$. The rather low potential barrier implies strong constraints on the maximal temperature in the early universe [29] as well as the Hubble parameter during inflation [30].

Stabilization of the compact dimension leaves the pseudoscalar partner of the radion, an axion, massless. Its coupling to non-Abelian gauge fields can then lead to a small mass. The contribution from QCD corrections can be expressed in terms of the pion mass and decay constant [31],

$$
m_{a} \sim \frac{m_{\pi} f_{\pi}}{M} \sim 10^{-10} \mathrm{eV}\left(\frac{10^{18} \mathrm{GeV}}{M}\right) .
$$

Depending on the cosmological initial conditions and evolution, coherent oscillations of such ultralight axions (and also the light radion) may yield an unacceptably large contribution to dark matter.

It is instructive to compare the described mechanism of radion stabilization with the approach of [20]. In both cases, supersymmetry is broken by a superpotential localized 
on a brane, and stabilization is achieved by the Casimir energy of massive and massless bulk fields. Here, a supersymmetric bulk mass $M_{\mathrm{bulk}} \simeq M_{\mathrm{GUT}} \gg m_{3 / 2}$ is induced by localized Fayet-Iliopoulos terms [14] via the Higgs mechanism. Finally, the brane field, which provides the non-zero superpotential, couples to massive bulk fields. This yields an additional contribution to the potential, which has the same order of magnitude as the Casimir energy [6]. In this way, a locally stable Minkowski or de Sitter vacuum can be obtained without the need of an additional uplifting mechanism.

\section{Conclusions}

We have considered five-dimensional supergravity theories compactified on the orbifold $S_{1} / \mathbb{Z}_{2}$. The expectation value of a chiral superfield localized at one of the fixed points, with non-vanishing superpotential, induces supersymmetry breaking by the radion field. The result is a no-scale model where the gravitino mass slides with the undetermined expectation value of the radion field. Perturbative corrections to the Kähler potential, Casimir energy and background values of bulk fields induced by localized Fayet-Iliopoulos terms, deform the no-scale model into an almost no-scale model. The size of the compact dimension is fixed at $L \sim 1 / M_{\text {bulk }} \sim 1 / M_{\text {GUT }}$.

Our study of five-dimensional orbifold supergravity models has been motivated by recent orbifold compactifications of the heterotic string which yield the supersymmetric standard model in four dimensions [28], with orbifold GUTs in five or six dimensions as intermediate step. It will be interesting to explicitly check whether the one-loop field theory corrections to the Kähler potential considered in this paper are indeed the leading part of the one-loop string corrections. This may be the case for anisotropic orbifold compactifications of the heterotic string leading to orbifold GUTs, since the Kaluza-Klein masses which contribute to the Casimir energy are smaller than the masses of string excitations. String theory also predicts a superpotential for localized chiral superfields and couplings of brane to bulk fields. Hence, also radion mediated supersymmetry breaking may be realized.

For a general perturbative correction $\kappa$ to the Kähler potential, we have calculated the correction to the effective radion potential to leading order in $\kappa$. The corresponding radion mass is volume suppressed compared to the gravitino mass. Moreover, since the stabilization is achieved by quantum corrections, the radion mass is also loop-suppressed. In addition, a tiny mass for the pseudoscalar partner of the radion, an axion, is generated by nonperturbative effects of non-Abelian gauge theories. Hence, the presence of light moduli fields is an unavoidable consequence of the proposed stabilization mechanism. This is in contrast to models where the nonperturbative dependence of the superpotential on moduli fields plays a crucial role. In such models the moduli fields can be heavy (cf. [12,32]).

It remains to be seen whether the light moduli predicted by our stabilization mechanism are consistent with the various potential 'cosmological moduli problems'. On the other hand, a radion with a mass two to four orders of magnitude smaller than the gravitino mass, could produce a distinctive signature in the cosmic gamma-ray spectrum and in this way become a 'smoking gun' for the existence of extra dimensions related to 
the scale of grand unification.

\section{Acknowledgement}

We would like to thank Laura Covi, Christian Gross, Jan Louis and Kai Schmidt-Hoberg for helpful discussions.

\section{References}

[1] E. Witten, Phys. Lett. B 155 (1985) 151.

[2] T. Appelquist and A. Chodos, Phys. Rev. D 28, 772 (1983).

[3] E. Ponton and E. Poppitz, JHEP 0106, 019 (2001). hep-ph/0105021.

[4] D. M. Ghilencea, D. Hoover, C. P. Burgess and F. Quevedo, JHEP 0509 (2005) 050 arXiv:hep-th/0506164.

[5] G. von Gersdorff and A. Hebecker, Phys. Lett. B 624 (2005) 270 arXiv:hep-th/0507131.

[6] W. Buchmuller, R. Catena and K. Schmidt-Hoberg, Nucl. Phys. B 804 (2008) 70 arXiv:0803.4501 [hep-ph]]; Nucl. Phys. B 821 (2009) 1 [arXiv:0902.4512 [hep-th]].

[7] H. M. Lee, H. P. Nilles and M. Zucker, Nucl. Phys. B 680 (2004) 177 arXiv:hep-th/0309195|, and references therein.

[8] K. Becker, M. Becker, M. Haack and J. Louis, JHEP 0206 (2002) 060 arXiv:hep-th/0204254.

[9] V. Balasubramanian, P. Berglund, J. P. Conlon and F. Quevedo, JHEP 0503 (2005) 007 arXiv:hep-th/0502058.

[10] M. Berg, M. Haack and B. Kors, Phys. Rev. Lett. 96 (2006) 021601 arXiv:hep-th/0508171.

[11] M. Cicoli, J. P. Conlon and F. Quevedo, JHEP 0810 (2008) 105 arXiv:0805.1029 [hep-th]].

[12] S. Kachru, R. Kallosh, A. Linde and S. P. Trivedi, Phys. Rev. D 68046005 (2003). arXiv:hep-th/0301240|.

[13] M. A. Luty and R. Sundrum, Phys. Rev. D 62 (2000) 035008 arXiv:hep-th/9910202.

[14] W. Buchmuller, C. Ludeling and J. Schmidt, JHEP 0709 (2007) 113 arXiv:0707.1651 [hep-ph]]. 
[15] D. Marti and A. Pomarol, Phys. Rev. D 64 (2001) 105025 |arXiv:hep-th/0106256|; Phys. Rev. D 66 (2002) 125005 |arXiv:hep-ph/0205034|.

[16] F. Paccetti Correia, M. G. Schmidt and Z. Tavartkiladze, Nucl. Phys. B 709 (2005) 141 |arXiv:hep-th/0408138; ; Nucl. Phys. B 763 (2007) 247 |arXiv:hep-th/0608058|.

[17] R. Brustein and S. P. de Alwis, Phys. Rev. D 69 (2004) 126006 |arXiv:hep-th/0402088|.

[18] M. Gomez-Reino and C. A. Scrucca, JHEP 0605 (2006) 015 |arXiv:hep-th/0602246|.

[19] L. Covi, M. Gomez-Reino, C. Gross, J. Louis, G. A. Palma and C. A. Scrucca, JHEP 0806 (2008) 057 arXiv:0804.1073 [hep-th]].

[20] M. A. Luty and N. Okada, JHEP 0304 (2003) 050 [arXiv:hep-th/0209178].

[21] A. Falkowski, JHEP 0505 (2005) 073 arXiv:hep-th/0502072|.

[22] E. Cremmer, S. Ferrara, C. Kounnas and D. V. Nanopoulos, Phys. Lett. B 133 (1983) 61.

[23] R. Rattazzi, C. A. Scrucca and A. Strumia, Nucl. Phys. B 674 (2003) 171 arXiv:hep-th/0305184.

[24] J. Bagger and D. V. Belyaev, Phys. Rev. D 67 (2003) 025004 arXiv:hep-th/0206024.

[25] C. Gross and A. Hebecker, Nucl. Phys. B 821 (2009) 354 [arXiv:0812.4267 [hep-ph]].

[26] H. Abe, T. Higaki and T. Kobayashi, Prog. Theor. Phys. 109 (2003) 809 |arXiv:hep-th/0210025|.

[27] G. von Gersdorff, M. Quiros and A. Riotto, Nucl. Phys. B 689 (2004) 76 arXiv:hep-th/0310190|.

[28] For recent reviews and references, see H. P. Nilles, S. Ramos-Sanchez, M. Ratz and P. K. S. Vaudrevange, "From strings to the MSSM," Eur. Phys. J. C 59 (2009) 249 [arXiv:0806.3905 [hep-th]];

J. Schmidt, "Local Grand Unification in the Heterotic Landscape", Fortsch. Phys., DOI: 10.1002/prop.200900079 [arXiv:0906.5501 [hep-th]].

[29] W. Buchmuller, K. Hamaguchi, O. Lebedev and M. Ratz, Nucl. Phys. B 699 (2004) 292 arXiv:hep-th/0404168]; JCAP 0501 (2005) 004 arXiv:hep-th/0411109.

[30] R. Kallosh and A. Linde, JHEP 0412 (2004) 004 arXiv:hep-th/0411011.

[31] S. Weinberg, "The quantum theory of fields. Vol. 2: Modern applications," Cambridge, UK: Univ. Pr. (1996) $489 p$

[32] K. Choi, A. Falkowski, H. P. Nilles and M. Olechowski, Nucl. Phys. B 718 (2005) 113 arXiv:hep-th/0503216. 